\title{
Serum neurofilament light chain and postural instability/gait difficulty (PIGD) subtypes of Parkinson's disease in the MARK-PD study
}

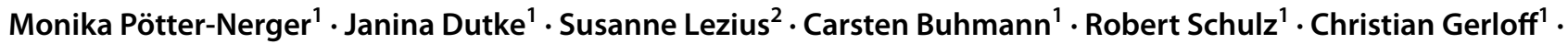 \\ Jens Kuhle ${ }^{3} \cdot$ Chi-un Choe' ${ }^{1}$
}

Received: 5 November 2021 / Accepted: 12 January 2022 / Published online: 24 January 2022

(c) The Author(s) 2022

\begin{abstract}
The PIGD (postural instability / gait difficulty) subtype of Parkinson's disease (PD) is associated with faster cognitive and motor decline. So far, there are no quantifiable biomarkers to aid clinical subtyping. Neurofilament light chain (NfL) is a highly specific marker of neuro-axonal damage and can be assessed in blood. Here, we investigated if serum NfL concentrations are associated with PIGD subtype and PIGD scores in PD patients at advanced disease stages. Furthermore, we evaluated if serum NfL is associated with motor and cognitive function assessed with MDS-UPDRS part III and Montreal cognitive assessment (MoCA). Serum NfL levels were analyzed with Single Molecule Assays (Simoa) in blood of 223 PD patients from the bioMARKers in Parkinson's Disease (MARK-PD) study. Serum NfL concentrations were higher in PIGD patients independent of age, sex and disease duration. In linear regression analysis, serum NfL levels were associated with MoCA, MDS-UPDRS III and PIGD scores in unadjusted models, but remained significant after adjustment only with PIGD scores. In conclusion, increased serum NfL levels were associated with PIGD subtype and PIGD scores in patients with advanced PD.
\end{abstract}

Keywords MDS-UPDRS $\cdot$ Hoehn and Yahr $\cdot$ MoCA $\cdot$ Biomarker

$\begin{array}{ll}\text { Abbreviations } & \\ \text { H\&Y } & \text { Hoehn and Yahr } \\ \text { LEDD } & \text { L-DOPA equivalent daily dose } \\ \text { NfL } & \text { Neurofilament light chain } \\ \text { NT-proBNP } & \text { N-terminal pro B-type natriuretic peptide } \\ \text { PD } & \text { Parkinson's disease } \\ \text { MOCA } & \text { Montreal cognitive assessment } \\ \text { MDS-UPDRS } & \text { Movement Disorders Society-Unified } \\ & \text { Parkinson's disease rating scale }\end{array}$

Chi-un Choe

cchoe@uke.de

1 Department of Neurology, University Medical Center Hamburg-Eppendorf, Martinistraße 52, 20246 Hamburg, Germany

2 Institute of Biometry and Medical Epidemiology, University Medical Center Hamburg-Eppendorf, Hamburg, Germany

3 Department of Neurology, University of Basel, Basel, Switzerland

\section{Introduction}

Postural and gait disturbances represent therapeutically demanding symptoms with high impact on quality of life in Parkinson's disease (PD). These axial symptoms are key features for one of the most used definitions of clinical motor subtypes of PD patients, which are classified into "PIGD" (Postural Instability / Gait Difficulty subtype), "TD" (Tremor Dominant subtype) or "Intermediate" subtypes (Jankovic et al. 1990; Stebbins et al. 2013). Cut-off values of the PIGD score, which are based on the MDS-UPDRS part III, define these PD subtypes. The PIGD score is also a continuous marker of axial symptoms and has prognostic and therapeutic implications (van Rooden et al. 2011). PIGD subtype patients exhibit a faster disease progression, a higher risk of cognitive decline, sleep disturbances, fatigue and autonomic dysfunction, an increased risk of freezing and falls, a shorter survival time and a worse quality of life compared with TD patients (Huang et al. 2019; Kwon et al. 2021; Lord et al. 2020; Ren et al. 2020a, b, 2021). Based on this phenomenological, symptom-orientated descriptive classification, motor subtypes change with disease progression and treatment (Luo et al. 2019). Therefore, more stable 
classification systems are warranted, which are based on the underlying pathophysiological differences and specified by additional biomarkers.

Blood-based biomarkers are easily accessible, quantifiable, objective parameters and can reflect the involved pathophysiological processes. Among these biomarkers, blood neurofilament light chain (NfL) has the potential to predict disease severity and duration as well as motor and cognitive decline (Hansson et al. 2017; Khalil et al. 2018; Lin et al. 2019; Mollenhauer et al. 2020; Niemann et al. 2021). Blood NfL levels reflect the degree of neuroaxonal damage. In early PD, NfL levels were higher in PIGD compared with TD patients after a 2 year follow-up, but not at baseline (Ng et al. 2020). Furthermore, higher NfL levels were associated with a worse cognitive and motor function and decline during follow-up at early stages ( $\mathrm{Ng}$ et al. 2020). However, the link between blood NfL with PIGD subtype and score in patients with advanced PD is unknown. Another recent study with 376 de novo PD patients from the PPMI database confirmed that higher serum NfL at baseline were associated with intense worsening of MDS-UPDRS III and PIGD scores, but not with progression of MoCA scores (Ye et al. 2021).

Here, we evaluated if serum NfL levels were associated with PIGD subtype and PIGD scores in advanced PD patients.

\section{Methods}

\section{Study design, ethical approval and patient consent}

The bioMARKers in Parkinson's disease (Mark-PD) study is a prospective observational single-center biobank at the University Medical Center Hamburg-Eppendorf, whose details have been described previously (Choe et al. 2020). The inclusion criteria were as follows: age $>18$ years, a clinical diagnosis of Parkinson's disease fulfilling Queen Square Brain Bank criteria. For this study, 223 PD patients were included with available clinical data to calculate PIGD scores and available serum NfL concentrations. The study protocol was approved by the Ethics Committee of the Hamburg Board of Physicians (PV5298). The investigation was conducted in accordance with the Declaration of Helsinki. Written informed consent was obtained from all participants.

\section{Clinical assessment}

Clinical assessments were performed as previously described (Choe et al. 2020). For this cross-sectional analysis, we used clinical assessments at baseline. Motor impairment was assessed using the Movement Disorder Society Unified Parkinson's disease Rating Scale part 3 (MDS-UPDRS III) in the $\mathrm{ON}$ medication and in case of deep brain stimulation $\mathrm{ON}$ stimulation state. Montreal cognitive assessment (MoCA) was used to assess cognitive function. H\&Y stage was recorded from medical records. Levodopa equivalent daily dose (LEDD) was calculated. Past medical history, including comorbidities (arterial hypertension, hypercholesterinemia, diabetes mellitus, prior stroke, prior myocardial infarction, heart failure, atrial fibrillation) and laboratory parameters (GFR) were documented from medical records. Dysautonomia was defined as diagnosis of orthostatic dysregulation, pathological Schellong-test or pathological MIGB-SPECT, as previously described (Choe et al. 2020). We calculated from the MDS-UPDRS the ratio of the tremor sum score (part II item 2.10, part III item 3.15-18) and the PIGD sum score (part II item 2.12, 2.13, Part III 3.10-3.12) (Stebbins et al. 2013). Accordingly, TD/PIGD scores $\leq 0.90$ classified PIGD subtypes, whereas TD/PIGD scores $>0.90$ were defined as non-PIGD. Motor and cognitive decline were defined as an increase of more than 4 points in the MDSUPDRS III and more than 2 points in the MoCA score during the follow-up period (Schwedhelm et al. 2021).

\section{Laboratory analysis}

The laboratory measurements were obtained from blood samples collected at baseline and processed as previously described (Choe et al. 2020). After centrifugation, serum samples were frozen at $-80{ }^{\circ} \mathrm{C}$ until biomarker analyses. Serum NfL levels were determined by single molecule array (Simoa) assay using the capture monoclonal antibody (mAB) 47:3 (initial dilution $0.3 \mathrm{mg} / \mathrm{mL}$; article number 27016) and the biotinylated detector mAB 2:1 $(0.1 \mu \mathrm{g} / \mathrm{mL}$; article number 27018) from UmanDiagnostic transferred onto the Simoa platform, as previously described (Niemann et al. 2021). The samples from the same participants were analyzed together in the same run to avoid within-subjects run-to-run variability. Intra- and interassay variability of the measurements were evaluated with three native serum samples in five consecutive runs on independent days. The mean coefficients of variation $(\mathrm{CVs})$ of duplicate determinations for concentration were $8.5 \%(9.5 \mathrm{pg} \mathrm{mL}-1$, sample 1), 5.4\% (23.2 pg mL - 1, sample 2) and 7.8\% (98.5 pg mL - 1, sample 3). Interassay CVs for serum were $7.8 \%$ (sample 1), 8.3\% (sample 2) and 4.9\% (sample 3).

\section{Statistical analysis}

Continuous variables are given as mean \pm standard deviation (SD) if normally distributed, otherwise as median [25th-75th percentile], and categorical variables are given as numbers (percentage) of participants. Relationships between continuous variables were assessed by Spearman's rank correlation (r). For linear regression analysis, NfL 
levels were $\log 2$-transformed. The association of continuous parameters with PD subtype (PIGD or non-PIGD) was assessed by linear regression models depicted by beta coefficients with corresponding 95\% confidence interval (CI) and $P$ - value are given. Linear regression analyses were performed unadjusted (model 1) and adjusted for age, sex and disease duration (model 2). $P$ values for comparison of 2 groups were obtained by Mann-Whitney- $U$-test for continuous and $X^{2}$ test for categorical variables, as appropriate.

Time to motor or cognitive decline was compared by log rank test and Kaplan-Meier curves were used for illustration. The following Cox Proportional Hazards models were used to examine the dependence of motor or cognitive decline: unadjusted (model 1) and adjusted for age, sex, disease duration and baseline MDS-UPDRS III or MoCA score, respectively (model 2). Presented are Hazard Ratios (HR) with corresponding $95 \% \mathrm{CI}$ and $P$ values.

All tests were two-sided and a $P$ value $<0.05$ was considered statistically significant. $P$ values were not adjusted for multiplicity due to the explorative character of the study.
Statistical analysis was performed with IBM SPSS Statistics (version 27, IBM Corp., Armonk, NY).

\section{Results}

\section{Patient characteristics}

In this Mark-PD sub-cohort, 223 PD patients were included with an average age of $65.5 \pm 9.3$ years, a disease duration of $11[6.5,15.5]$ years and $65 \%$ male (Table 1). Median NfL concentrations were 18.1 [12.6, 27.2] (Table 1). Among these patients, $73 \%$ were classified as PIGD subtypes according to PIGD scores. The median LEDD was $901[640,1263]$ $\mathrm{mg} / \mathrm{d}$, MDS-UPDRS III score was $23[17,31]$ and Hoehn and Yahr stage was $2[2,3]$.
Table 1 Baseline characteristics of non-PIGD and PIGD subtypes in Mark-PD

\begin{tabular}{|c|c|c|c|c|}
\hline Characteristics & $\begin{array}{l}\text { non-PIGD } \\
(n=61)\end{array}$ & $\begin{array}{l}\text { PIGD } \\
(n=162)\end{array}$ & $P$ value & $\begin{array}{l}\text { Mark-PD subcohort } \\
(n=223)\end{array}$ \\
\hline \multicolumn{5}{|l|}{ Demographic parameters } \\
\hline Age, years & $63.3(9.8)$ & $66.4(9.0)$ & $0.025^{*}$ & $65.5(9.3)$ \\
\hline Male & $45(73)$ & $101(62)$ & 0.110 & $146(65)$ \\
\hline Dysautonomia & $16(26)$ & $39(24)$ & 0.739 & $55(25)$ \\
\hline Hypertension & $29(48)$ & $61(38)$ & 0.180 & $90(40)$ \\
\hline Hyperlipidemia & $9(15)$ & $18(11)$ & 0.457 & $27(12)$ \\
\hline Diabetes & $9(15)$ & $13(8)$ & 0.133 & $22(10)$ \\
\hline Prior myocardial infarct & $6(10)$ & $13(8)$ & 0.666 & $19(9)$ \\
\hline Prior stroke & $3(5)$ & $9(6)$ & 0.851 & $12(5)$ \\
\hline Congestive heart failure & $4(7)$ & $5(3)$ & 0.240 & $9(4)$ \\
\hline Atrial fibrillation & $3(5)$ & $13(8)$ & 0.423 & $16(7)$ \\
\hline \multicolumn{5}{|l|}{ Laboratory parameters } \\
\hline $\mathrm{CRP}, \mathrm{mg} / \mathrm{dl}$ & $0.53[1.10,2.55]$ & $1.33[0.62,2.94]$ & 0.186 & $1.2[0.6,2.9]$ \\
\hline Creatinine, $\mathrm{mg} / \mathrm{dl}$ & $0.81[0.71,0.89]$ & $0.77[0.67,0.89]$ & 0.121 & $0.78[0.68,0.88]$ \\
\hline $\mathrm{Nfl}, \mathrm{pg} / \mathrm{ml}$ & $15.5[9.8,22.1]$ & $19.2[13.9,33.2]$ & $<0.001 * * *$ & $18.1[12.6,27.2]$ \\
\hline \multicolumn{5}{|l|}{ Neurological parameters } \\
\hline MDS-UPDRS III, points & $23[17,30]$ & $23[16,32]$ & 0.830 & $23[17,31]$ \\
\hline Hoehn and Yahr, stage & $2[2,2.5]$ & $2[2,3]$ & 0.290 & $2[2,3]$ \\
\hline Motor fluctuations & $30(50)$ & $111(69)$ & $0.011^{*}$ & 141 \\
\hline LEDD, mg/d & $672[430,938]$ & $1005[734,1379]$ & $<0.001 * * *$ & $901[640,1263]$ \\
\hline MoCA, points & $27[25,29]$ & $26[23,28]$ & $0.001 * *$ & $27[24,28]$ \\
\hline Disease duration, years & $8.5[4,13]$ & $12[8,17]$ & $0.001 * *$ & $11[6.5,15.5]$ \\
\hline DBS & $14(23)$ & $63(39)$ & $0.026^{*}$ & $77(35)$ \\
\hline
\end{tabular}

Data are mean (SD), $n(\%)$ or median [IQR], as appropriate. Categorical variables are given as numbers (percentages) of participants. Comparisons between non-PIGD and PIGD subtypes were analyzed with $t$ test, Mann-Whitney test or Chi squared test, as appropriate $\left({ }^{*} P<0.05, * * P<0.01\right.$, $\left.* * * P<0.001\right)$.

$C R P$ C-reactive protein; $N f$ neurofilament light chain; UPDRS unified Parkinson's disease ranking scale; $L E D D$ L-dopa equivalence dose; MoCA Montreal cognitive assessment; $D B S$ deep brain stimulation PIGD postural instability / gait difficulty 


\section{Clinical and laboratory parameters of PIGD and non-PIGD patients}

Compared with non-PIGD patients, PIGD patients had a longer disease duration, higher LEDD, lower MoCA scores, more often motor fluctuations and DBS treatment (Table 1). Among laboratory parameters, PIGD patients had increased serum NfL concentrations. In unadjusted and adjusted regression models, differences between non-PIGD and PIGD patients remained significant for MoCA score and serum NfL, whereas MDS-UPDRS III scores did not significantly differ between PIGD and nonPIGD patients (Table 2). Similar results were obtained after excluding PD patients with DBS (Supplementary Table 2).

\section{Association of serum NfL with MoCA, MDS-UPDRS III and PIGD scores}

Besides differentiation of non-PIGD and PIGD patients, we evaluated the continuous associations of serum NfL with clinical and laboratory parameters. In correlation analysis, serum NfL revealed significant relationships with age, MDS-UPDRS III, LEDD, MoCA and PIGD score (Supplementary Table 1). Although serum NfL was significantly associated with MoCA and MDS-UPDRS III scores in unadjusted linear regression analysis, these associations did not remain significant after adjustment for age, sex and disease duration (Table 3). In contrast, serum NfL concentrations were significantly associated with PIGD score in unadjusted regression models and remained significant after adjustment (Table 3). Similar results were obtained after excluding PD patients with DBS, underlining the robustness of this finding (Supplementary Table 3).

\section{Discussion}

Our main findings were that in PD patients with advanced disease (1) serum NfL levels were increased in PIGD subtype (2) PIGD patients had worse MoCA, but similar MDSUPDRS III total scores compared with non-PIGD patients and (3) increased serum NfL concentrations were incrementally associated with higher PIGD score.

In PD patients, increased blood NfL levels were correlated with worse motor function in a Taiwanese cohort, but not in a Dutch study (Lin et al. 2019; Oosterveld et al. 2020). Here, we did not observe an association of serum NfL with MDS-UPDRS III scores independent of age, sex and disease duration. However, the PIGD score, which is based on MDS-UPDRS III items focussing on postural instability and gait disturbance, revealed a significant association with serum NfL. This finding is in line with a recent study reporting increased serum NfL levels in PIGD patients at earlier disease stages ( $\mathrm{Ng}$ et al. 2020). Furthermore, increased $\mathrm{NfL}$ levels predicted a more intense worsening of the PIGD, but not tremor score (Ye et al. 2021). These findings suggest that a more pronounced neuro-axonal damage underlies PD symptoms of postural instability / gait difficulty. A faster motor and cognitive decline, which are found in PIGD patients, might therefore be consequences of more severe neurodegenerative processes.

Table 2 Linear regression analysis of PIGD subtype with MDS-UPDRS III, MoCA and serum NfL

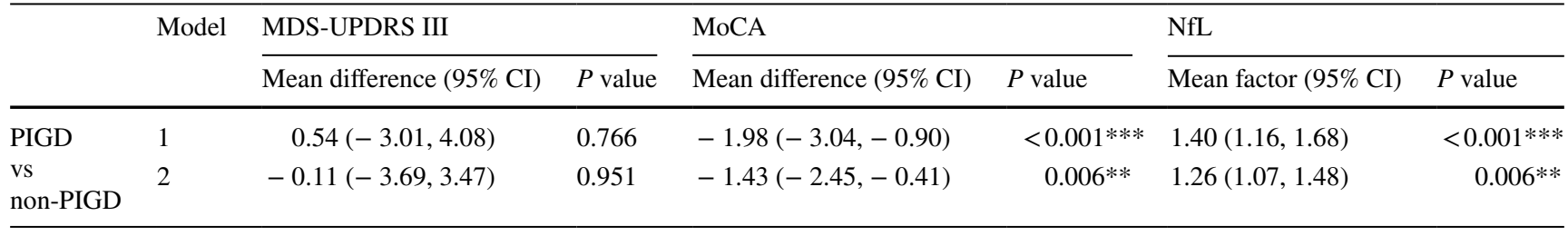

ANCOVA with $\beta$ coefficients and 95\% confidence interval (model 1: unadjusted; model 2: adjusted for age, sex and disease duration)

Table 3 Linear regression analysis of serum NfL with MDS-UPDRS III, MoCA and PIGD score

\begin{tabular}{|c|c|c|c|c|c|c|c|}
\hline & \multirow[t]{2}{*}{ Model } & \multicolumn{2}{|l|}{ MoCA } & \multicolumn{2}{|l|}{ MDS-UPDRS III } & \multicolumn{2}{|l|}{ PIGD score } \\
\hline & & $\begin{array}{l}\text { Mean difference } \\
(95 \% \mathrm{CI})\end{array}$ & $P$ value & $\begin{array}{l}\text { Mean difference } \\
(95 \% \mathrm{CI})\end{array}$ & $P$ value & $\begin{array}{l}\text { Mean difference } \\
(95 \% \mathrm{CI})\end{array}$ & $P$ value \\
\hline \multirow{2}{*}{$\begin{array}{l}\text { NfL } \\
\text { (per twofold increase) }\end{array}$} & 1 & $-1.19(-1.70,-0.67)$ & $<0.001 * * *$ & $2.46(0.77,4.14)$ & $0.004 * *$ & $0.25(0.14,0.36)$ & $<0.001 * * *$ \\
\hline & 2 & $-0.45(-1.03,0.13)$ & 0.129 & $1.25(-0.74,3.25)$ & 0.217 & $0.15(0.02,0.28)$ & $0.022 *$ \\
\hline
\end{tabular}

Linear regression analysis with $\beta$ coefficients and 95\% confidence interval (model 1: unadjusted; model 2: adjusted for age, sex and disease duration) 
In contrast to motor function, the Taiwanese and Dutch study revealed consistent associations of high blood NfL levels with cognitive impairment (Lin et al. 2019; Oosterveld et al. 2020). Although we also observed a correlation between serum NfL with MoCA scores, this association was not independent of age, sex and disease duration. Compared with the Mini-Mental-State-Examination (MMSE) used in previously published studies, we used the MoCA test, which is known to be more sensitive for cognitive deficits. Therefore, serum NfL might be less sensitive to detect milder cognitive changes. Furthermore, median disease durations were much shorter in the Dutch and Taiwanese study compared with PD patients in Mark-PD. It is therefore possible, that the predictive value of serum NfL changes during the course or disease progression.

Surprisingly, baseline NfL levels did not differ between PIGD and tremor subtypes at early disease stages, whereas increased levels were found in PIGD subtypes after 2 years of follow-up in a PD cohort from Singapore ( $\mathrm{Ng}$ et al. 2020). In line with these findings, serum NfL levels were significantly higher in PIGD patients at advanced disease stages in our cohort. Although the PIGD subtype is based on a phenomenological classification, neurochemical and neuropathological findings suggest a more severe PD pathology. Lower amyloid-beta 42 (A $\beta 1-42)$ and phospho-tau 181 concentrations in the CSF were associated with PIGD subtype indicating a higher degree of neurodegeneration (Kang et al. 2013a, b). Similarly, Lewy bodies and amyloid-beta plaques were more abundant in the cortex of PIGD subtypes suggestive of a more severe pathology (Selikhova et al. 2009). Most importantly, these neurochemical and neuropathological findings correlated with a faster motor decline in PIGD patients. Our study suggests that in PD patients with a median disease duration of 11 years, the extent of neurodegeneration is still significantly different between PIGD and non-PIGD patients. Even more important, incrementally increasing serum NfL levels revealed a robust association with PIGD symptoms and scores, which indicate a continuous spectrum of neurodegeneration. The PIGD subtype is therefore more a continuum than a clear cut entity (Kotagal 2016).

A limitation of our study is the cross-sectional, singlecenter, hospital-based study design, which does not allow to draw causal relationships. Although we present prospective data on a subset of patients, the size of this sub-cohort is very small and does not allow adjustment of multiple covariates.

In conclusion, we propose that serum NfL remains a valid biomarker to distinguish PIGD subtype in advanced PD patients and allows an incremental quantification of PIGD symptoms.
Supplementary Information The online version contains supplementary material available at https://doi.org/10.1007/s00702-022-02464-x.

Author contributions (1) Research project: A. Conception of MarkPD: CUC. Conception of this substudy: MP and CUC. B. Organization: MP, JD, CB, JK, CUC. C. Execution: MP, JD, CB, JK, CUC. (2) Statistical Analysis: A. Design: MP, SL, RS, CUC. B. Execution: SL, CUC. C. Review and Critique: MP, JD, SL, CB, CG, RS, JK, CUC. (3) Manuscript: A. Writing of the first draft: MP, JK, CUC. B. Review and Critique: MP, JD, SL, CB, CG, RS, JK, CUC. All authors have contributed to, seen and approved the manuscript.

Funding Open Access funding enabled and organized by Projekt DEAL. Dr. Choe was supported by an Else Kröner Exzellenzstipendium from the Else Kröner-Fresenius Stiftung during the conduct of the study (grant number: 2018_EKES.04). Dr. Pötter-Nerger was supported by grant from the Deutsche Forschungsgemeinschaft (DFG) SFB 936, C8.

\section{Declarations}

Conflict of interest JD, SL and TZ report no disclosures. MPN reports personal fees from Abbvie, Abbott and study fees from Boston Scientific, Licher, Abbott outside the submitted work. CB received a grant from the Georg \& Jürgen Rickertsen Stiftung Hamburg, served on the scientific advisory boards for Bial and Zambon and received honoraria for lectures from Abbvie, Bial, GE Healthcare, Licher, Orion Pharma and UCB Pharma. CG reports personal fees from AMGEN, Boehringer Ingelheim, from Daiichi Sankyo, Abbott, Prediction Biosciences, Novartis and Bayer outside the submitted work. CUC reports personal fees from Pfizer and Zambon outside the submitted work.

Open Access This article is licensed under a Creative Commons Attribution 4.0 International License, which permits use, sharing, adaptation, distribution and reproduction in any medium or format, as long as you give appropriate credit to the original author(s) and the source, provide a link to the Creative Commons licence, and indicate if changes were made. The images or other third party material in this article are included in the article's Creative Commons licence, unless indicated otherwise in a credit line to the material. If material is not included in the article's Creative Commons licence and your intended use is not permitted by statutory regulation or exceeds the permitted use, you will need to obtain permission directly from the copyright holder. To view a copy of this licence, visit http://creativecommons.org/licenses/by/4.0/.

\section{References}

Choe CU, Niemann L, Englisch C, Petersen E, Buhmann C, PotterNerger M, Blankenberg S, Gerloff C, Schwedhelm E, Zeller T (2020) Subclinical cardiac microdamage, motor severity, and cognition in Parkinson's Disease. Mov Disord 35(10):1863-1868. https://doi.org/10.1002/mds.28129

Hansson O, Janelidze S, Hall S, Magdalinou N, Lees AJ, Andreasson U, Norgren N, Linder J, Forsgren L, Constantinescu R, Zetterberg H, Blennow K, Fs SB (2017) Blood-based NfL: a biomarker for differential diagnosis of parkinsonian disorder. Neurology 88(10):930-937. https://doi.org/10.1212/WNL.0000000000 003680

Huang X, Ng SY, Chia NS, Setiawan F, Tay KY, Au WL, Tan EK, Tan LC (2019) Non-motor symptoms in early Parkinson's disease with different motor subtypes and their associations with quality of life. Eur J Neurol 26(3):400-406. https://doi.org/10.1111/ene.13803 
Jankovic J, McDermott M, Carter J, Gauthier S, Goetz C, Golbe L, Huber S, Koller W, Olanow C, Shoulson I et al (1990) Variable expression of Parkinson's disease: a base-line analysis of the DATATOP cohort. Parkinson Study Grp Neurol 40(10):1529_ 1534. https://doi.org/10.1212/wnl.40.10.1529

Kang JH, Irwin DJ, Chen-Plotkin AS, Siderowf A, Caspell C, Coffey CS, Waligorska T, Taylor P, Pan S, Frasier M, Marek K, Kieburtz K, Jennings D, Simuni T, Tanner CM, Singleton A, Toga AW, Chowdhury S, Mollenhauer B, Trojanowski JQ, Shaw LM, Parkinson's Progression Markers I (2013a) Association of cerebrospinal fluid beta-amyloid 1-42, T-tau, P-tau181, and alphasynuclein levels with clinical features of drug-naive patients with early Parkinson disease. JAMA Neurol 70(10):1277-1287. https:// doi.org/10.1001/jamaneurol.2013.3861

Kang L, Janowska MK, Moriarty GM, Baum J (2013b) Mechanistic insight into the relationship between $\mathrm{N}$-terminal acetylation of alpha-synuclein and fibril formation rates by NMR and fluorescence. PLoS ONE 8(9):e75018. https://doi.org/10.1371/journal. pone. 0075018

Khalil M, Teunissen CE, Otto M, Piehl F, Sormani MP, Gattringer T, Barro C, Kappos L, Comabella M, Fazekas F, Petzold A, Blennow K, Zetterberg H, Kuhle J (2018) Neurofilaments as biomarkers in neurological disorders. Nat Rev Neurol 14(10):577-589. https:// doi.org/10.1038/s41582-018-0058-z

Kotagal V (2016) Is PIGD a legitimate motor subtype in Parkinson disease? Ann Clin Transl Neurol 3(6):473-477. https://doi.org/ 10.1002/acn3.312

Kwon KY, Lee EJ, Lee M, Ju H, Im K (2021) Impact of motor subtype on non-motor symptoms and fall-related features in patients with early Parkinson's disease. Geriatr Gerontol Int 21(5):416-420. https://doi.org/10.1111/ggi.14156

Lin CH, Li CH, Yang KC, Lin FJ, Wu CC, Chieh JJ, Chiu MJ (2019) Blood NfL: A biomarker for disease severity and progression in Parkinson disease. Neurology 93(11):e1104-e1111. https://doi. org/10.1212/WNL.0000000000008088

Lord SR, Bindels H, Ketheeswaran M, Brodie MA, Lawrence AD, Close JCT, Whone AL, Ben-Shlomo Y, Henderson EJ (2020) Freezing of gait in people with Parkinson's disease: nature, occurrence, and risk factors. J Parkinsons Dis 10(2):631-640. https:// doi.org/10.3233/JPD-191813

Luo L, Andrews H, Alcalay RN, Poyraz FC, Boehme AK, Goldman JG, Xie T, Tuite P, Henchcliffe C, Hogarth P, Amara AW, Frank S, Sutherland M, Kopil C, Naito A, Kang UJ (2019) Motor phenotype classification in moderate to advanced PD in BioFIND study. Parkinsonism Relat Disord 65:178-183. https://doi.org/10.1016/j. parkreldis.2019.06.017

Mollenhauer B, Dakna M, Kruse N, Galasko D, Foroud T, Zetterberg H, Schade S, Gera RG, Wang W, Gao F, Frasier M, Chahine LM, Coffey CS, Singleton AB, Simuni T, Weintraub D, Seibyl J, Toga AW, Tanner CM, Kieburtz K, Marek K, Siderowf A, Cedarbaum JM, Hutten SJ, Trenkwalder C, Graham D (2020) Validation of serum neurofilament light chain as a biomarker of Parkinson's disease progression. Mov Disord 35(11):1999-2008. https://doi. org/10.1002/mds.28206

Ng ASL, Tan YJ, Yong ACW, Saffari SE, Lu Z, Ng EY, Ng SYE, Chia NSY, Choi X, Heng D, Neo S, Xu Z, Keong NCH, Tay KY, Au WL, Tan LCS, Tan EK (2020) Utility of plasma neurofilament light as a diagnostic and prognostic biomarker of the postural instability gait disorder motor subtype in early Parkinson's disease. Mol Neurodegener 15(1):33. https://doi.org/10. 1186/s13024-020-00385-5

Niemann L, Lezius S, Maceski A, Leppert D, Englisch C, Schwedhelm E, Zeller T, Gerloff C, Kuhle J, Choe CU (2021) Serum neurofilament is associated with motor function, cognitive decline and subclinical cardiac damage in advanced Parkinson's disease (MARK-PD). Parkinsonism Relat Disord 90:44-48

Oosterveld LP, Verberk IMW, Majbour NK, El-Agnaf OM, Weinstein HC, Berendse HW, Teunissen CE, van de Berg WDJ (2020) CSF or serum neurofilament light added to alpha-Synuclein panel discriminates Parkinson's from controls. Mov Disord 35(2):288-295. https://doi.org/10.1002/mds.27897

Ren J, Hua P, Li Y, Pan C, Yan L, Yu C, Zhang L, Xu P, Zhang M, Liu W (2020a) Comparison of three motor subtype classifications in de novo Parkinson's disease patients. Front Neurol 11:601225. https://doi.org/10.3389/fneur.2020.601225

Ren J, Hua P, Pan C, Li Y, Zhang L, Zhang W, Xu P, Zhang M, Liu W (2020b) Non-motor symptoms of the postural instability and gait difficulty subtype in de novo Parkinson's Disease patients: a cross-sectional study in a single center. Neuropsychiatr Dis Treat 16:2605-2612. https://doi.org/10.2147/NDT.S280960

Ren J, Pan C, Li Y, Li L, Hua P, Xu L, Zhang L, Zhang W, Xu P, Liu W (2021) Consistency and stability of motor subtype classifications in patients with de novo Parkinson's disease. Front Neurosci 15:637896. https://doi.org/10.3389/fnins.2021.637896

Schwedhelm E, Englisch C, Niemann L, Lezius S, von Lucadou M, Marmann K, Boger R, Peine S, Daum G, Gerloff C, Choe CU (2021) Sphingosine-1-phosphate, motor severity, and progression in Parkinson's disease (MARK-PD). Mov Disord. https://doi.org/ $10.1002 / \mathrm{mds} .28652$

Selikhova M, Williams DR, Kempster PA, Holton JL, Revesz T, Lees AJ (2009) A clinico-pathological study of subtypes in Parkinson's disease. Brain 132(Pt 11):2947-2957. https://doi.org/10. 1093/brain/awp234

Stebbins GT, Goetz CG, Burn DJ, Jankovic J, Khoo TK, Tilley BC (2013) How to identify tremor dominant and postural instability/ gait difficulty groups with the movement disorder society unified Parkinson's disease rating scale: comparison with the unified Parkinson's disease rating scale. Mov Disord 28(5):668-670. https:// doi.org/10.1002/mds. 25383

van Rooden SM, Colas F, Martinez-Martin P, Visser M, Verbaan D, Marinus J, Chaudhuri RK, Kok JN, van Hilten JJ (2011) Clinical subtypes of Parkinson's disease. Mov Disord 26(1):51-58. https:// doi.org/10.1002/mds.23346

Ye R, Locascio JJ, Goodheart AE, Quan M, Zhang B, Gomperts SN (2021) Serum NFL levels predict progression of motor impairment and reduction in putamen dopamine transporter binding ratios in de novo Parkinson's disease: an 8-year longitudinal study. Parkinsonism Relat Disord 85:11-16. https://doi.org/10.1016/j. parkreldis.2021.02.008

Publisher's Note Springer Nature remains neutral with regard to jurisdictional claims in published maps and institutional affiliations. 